Supporting Information for

\title{
Highly Potent and Selective Histone Deacetylase 6 Inhibitors Designed Based on a Small-molecular Substrate
}

\author{
Takayoshi Suzuki, ${ }^{* \dagger}$ Akiyasu Kouketsu, ${ }^{\dagger}$ Yukihiro Itoh,${ }^{\dagger}$ Shinya Hisakawa, ${ }^{\dagger}$ Satoko Maeda, ${ }^{\ddagger}$ Minoru \\ Yoshida, ${ }^{\ddagger \S}$ Hidehiko Nakagawa, ${ }^{\dagger}$ and Naoki Miyata ${ }^{*}{ }^{\dagger}$ \\ ${ }^{\dagger}$ Graduate School of Pharmaceutical Sciences, Nagoya City University, 3-1 Tanabe-dori, Mizuho-ku, Nagoya, \\ Aichi 467-8603, Japan \\ ${ }^{\ddagger}$ RIKEN, Saitama 351-0198, Japan \\ ${ }^{\S}$ CREST Research Project, Japan Science and Technology Agency, Saitama 332-001, Japan
}

\section{Experimental}

\section{Chemistry}

General Methods. Melting points were determined using a Yanagimoto micro melting point apparatus or a Büchi 545 melting point apparatus and were not corrected. Proton nuclear magnetic resonance spectra $\left({ }^{1} \mathrm{H}\right.$ NMR) and carbon nuclear magnetic resonance spectra $\left({ }^{13} \mathrm{C}\right.$ NMR) were recorded on a JEOL JNM-LA500 spectrometer in $\mathrm{CDCl}_{3}$ as the solvent. Chemical shifts $(\delta)$ were reported in parts per million relative to the internal standard tetramethylsilane. Elemental analysis was performed with a Yanaco CHN CORDER NT-5 analyzer, and all values were within $\pm 0.4 \%$ of the calculated values. High-resolution mass spectra (HRMS) were recorded on a JEOL JMS-SX102A mass spectrometer. All reagents and solvents were purchased from Aldrich, Tokyo Kasei Kogyo, Wako Pure Chemical Industries, or Kanto Kagaku, and were used without 
purification. Flash column chromatography was performed using Silica Gel 60 (particle size 0.046-0.063 mm) supplied by Merck.

(S)-Thioisobutyric acid $S$-[6-tert-butoxycarbonylamino-6-(4-trifluoromethyl-2-oxo-2H-chromen-7ylcarbamoyl)hexyl] ester (12b)

Step 1: Preparation of (S)-7-Bromo-2-tert-butoxycarbonylaminoheptanoic acid (22). To an aqueous solution (45 mL) of (S)-2-amino-7-bromoheptanoic acid (21) $(1.08 \mathrm{~g}, 4.90 \mathrm{mmol})$ and $\mathrm{Et}_{3} \mathrm{~N}(2 \mathrm{~mL})$ was added a solution of $(\mathrm{Boc})_{2} \mathrm{O}(2.05 \mathrm{~g}, 9.40 \mathrm{mmol})$ in $\mathrm{THF}(50 \mathrm{~mL})$ and the mixture was stirred overnight at room temperture. The reaction mixture was poured into $2 \mathrm{~N}$ aqueous $\mathrm{NaOH}(15 \mathrm{~mL})$, and the whole was washed with AcOEt. To the separated aqueous layer was added $10 \%$ citric acid $(15 \mathrm{~mL})$, and the whole was extracted with AcOEt. The organic layer was washed with water and brine, and dried over $\mathrm{Na}_{2} \mathrm{SO}_{4}$. Filtration and concentration in vacuo gave $1.33 \mathrm{~g}(94 \%)$ of 22 as a colorless oil: ${ }^{1} \mathrm{H}-\mathrm{NMR}\left(\mathrm{CDCl}_{3}, 500 \mathrm{MHz}, \delta\right.$; ppm) 5.02 $(1 \mathrm{H}, \mathrm{d}, J=7.0 \mathrm{~Hz}), 4.31(1 \mathrm{H}, \mathrm{s}), 3.40(2 \mathrm{H}, \mathrm{t}, J=6.7 \mathrm{~Hz}), 1.87(1 \mathrm{H}, \mathrm{m}), 1.72-1.65(1 \mathrm{H}, \mathrm{m}), 1.87(2 \mathrm{H}$, quintet, $J$ $=7.1 \mathrm{~Hz}), 1.52-1.43(13 \mathrm{H}, \mathrm{m})$.

Step 2: Preparation of $(S)$-[6-Bromo-1-(2-oxo-4-trifluoromethyl-2H-chromen-7-ylcarbamoyl)hexyl] carbamic acid tert-butyl ester (23d). To a solution of 7-amino-4-(trifluoromethyl)coumarin (688 mg, 3.00 mmol) and 22 (973 mg, $3.00 \mathrm{mmol})$ obtained above in dry pyridine (18 $\mathrm{mL})$ was added phosphoryl chloride $(0.75 \mathrm{~mL}, 2.97 \mathrm{mmol})$ at $-15^{\circ} \mathrm{C}$, and the solution was stirred at $-15^{\circ} \mathrm{C}$ for $15 \mathrm{~min}$. The solution was poured into water, and the whole was extracted with AcOEt. The AcOEt layer was separated, washed with saturated aqueous $\mathrm{NaHCO}_{3}, 10 \%$ aqueous citric acid and brine, then dried over $\mathrm{Na}_{2} \mathrm{SO}_{4}$. Filtration, concentration in vacuo and purification by silica gel flash column chromatography $(\mathrm{AcOEt} / n$-hexane $=1 / 3)$ gave $922 \mathrm{mg}(57$ $\%)$ of $23 d$ as a white solid: ${ }^{1} \mathrm{H}-\mathrm{NMR}\left(\mathrm{CDCl}_{3}, 500 \mathrm{MHz}, \delta\right.$; ppm) $9.02(1 \mathrm{H}, \mathrm{s}), 7.80(1 \mathrm{H}, \mathrm{d}, J=1.8 \mathrm{~Hz}), 7.62$ $(1 \mathrm{H}, \mathrm{d}, J=7.9 \mathrm{~Hz}), 7.46(1 \mathrm{H}, \mathrm{d}, J=9.1 \mathrm{~Hz}), 6.67(1 \mathrm{H}, \mathrm{s}), 4.97(1 \mathrm{H}, \mathrm{s}), 4.19(1 \mathrm{H}, \mathrm{s}), 3.41(2 \mathrm{H}, \mathrm{t}, J=6.7 \mathrm{~Hz})$ 2.02-1.96 (1H, m), $1.88(2 \mathrm{H}$, quintet, $J=7.0 \mathrm{~Hz}), 1.71-1.60(1 \mathrm{H}, \mathrm{m}), 1.48(9 \mathrm{H}, \mathrm{s}), 1.52-1.35(4 \mathrm{H}, \mathrm{m})$.

Step 3: Preparation of (S)-Thioisobutyric acid S-[6-tert-butoxycarbonylamino-6-(4-trifluoromethyl -2-oxo-2H-chromen-7-ylcarbamoyl)hexyl] ester (12b). To a solution of 23d (443 mg, 0.920 mmol) obtained 
above in $\mathrm{EtOH}(10 \mathrm{~mL})$ was added a solution of thioisobutyric acid $(455 \mathrm{mg}, 4.38 \mathrm{mmol})$ in $\mathrm{EtOH}(5 \mathrm{~mL})$ and triethylamine $(1 \mathrm{~mL}, 13.6 \mathrm{mmol})$ and the mixture was stirred overnight at room temperature. The reaction mixture was poured into water and the whole was extracted with AcOEt. The AcOEt layer was separated, washed with $10 \%$ aqueous citric acid and brine, and dried over $\mathrm{Na}_{2} \mathrm{SO}_{4}$. Filtration, concentration in vacuo, purification by silica gel flash column chromatography $(\mathrm{AcOE} / n$-hexane $=1 / 4)$ and recrystallization from AcOEt/ $n$-hexane gave $250 \mathrm{mg}$ (54\%) of $\mathbf{1 2 b}$ as white crystals: mp $163-165{ }^{\circ} \mathrm{C} ;{ }^{1} \mathrm{H}-\mathrm{NMR}\left(\mathrm{CDCl}_{3}, 500 \mathrm{MHz}\right.$, $\delta$; ppm) ) $9.07(1 \mathrm{H}, \mathrm{s}), 7.81(1 \mathrm{H}, \mathrm{s}), 7.62(1 \mathrm{H}, \mathrm{d}, J=7.9 \mathrm{~Hz}), 7.41(1 \mathrm{H}, \mathrm{d}, J=7.3 \mathrm{~Hz}), 6.68(1 \mathrm{H}, \mathrm{s}), 5.06(1 \mathrm{H}, \mathrm{s})$, $4.18(1 \mathrm{H}$, broad s), $2.84(2 \mathrm{H}, \mathrm{t}, J=7.8 \mathrm{~Hz}), 2.73(1 \mathrm{H}$, quintet, $J=6.9 \mathrm{~Hz}), 1.99-1.95(1 \mathrm{H}, \mathrm{m}), 1.70-1.65(1 \mathrm{H}$, m), 1.60-1.54 (2H, m), $1.47(9 \mathrm{H}, \mathrm{s}), 1.45-1.40(4 \mathrm{H}, \mathrm{m}), 1.18(6 \mathrm{H}, \mathrm{d}, \mathrm{J}=7.0 \mathrm{~Hz}) ;{ }^{13} \mathrm{C}-\mathrm{NMR}\left(\mathrm{CDCl}_{3}, 500 \mathrm{MHz}\right.$, $\delta$; ppm) 204.59, 171.00, 159.10, 155.24, 142.34, 128.50, 125.83, 122.40, 116.18, 113.84, 109.36, 107.51, 81.26, 77.24, 43.14, 29.32, 28.31, 28.20, 25.12, 19.42; Anal. Calcd. for $\mathrm{C}_{20} \mathrm{H}_{36} \mathrm{~N}_{2} \mathrm{O}_{6} \mathrm{~S} \cdot 1 / 4 \mathrm{H}_{2} \mathrm{O}: \mathrm{C}, 55.46 ; \mathrm{H}$, 6.00; N, 4.97. Found: C, 55.21; H, 5.81; N, 5.21.

Compounds 13b-16b were prepared from 22 and the corresponding amines using the procedure described for $12 b$.

(S)-Thioisobutyric acid $S$-(6-tert-butoxycarbonylamino-6-phenylcarbamoylhexyl) ester (13b): yellow oil; ${ }^{1} \mathrm{H}-\mathrm{NMR}\left(\mathrm{CDCl}_{3}, 500 \mathrm{MHz}, \delta\right.$; ppm) $8.22(1 \mathrm{H}, \mathrm{s}), 7.52(2 \mathrm{H}, \mathrm{d}, J=8.0 \mathrm{~Hz}), 7.31(2 \mathrm{H}, \mathrm{t}, J=7.8), 7.10(1 \mathrm{H}$, $\mathrm{t}, J=7.5 \mathrm{~Hz}), 5.04(1 \mathrm{H}, \mathrm{s}), 4.17(1 \mathrm{H}, \mathrm{s}), 2.84(2 \mathrm{H}, \mathrm{t}, J=7.3 \mathrm{~Hz}), 2.73(1 \mathrm{H}$, quintet, $J=7.0 \mathrm{~Hz}), 1.96-1.92(1 \mathrm{H}$, m), 1.65-1.55 (3H, m), 1.50-1.45 (13H, m), $1.19(6 \mathrm{H}, \mathrm{d}, J=7.0 \mathrm{~Hz}) ;{ }^{13} \mathrm{C}-\mathrm{NMR}\left(\mathrm{CDCl}_{3}, 500 \mathrm{MHz}, \delta ; \mathrm{ppm}\right)$ 204.45, 170.26, 129.00, 124.38, 119.87, 77.22, 43.12, 29.36, 28.31, 28.25, 25.13, 19.42; HRMS Calcd. for $\mathrm{C}_{22} \mathrm{H}_{34} \mathrm{O}_{4} \mathrm{~N}_{2} \mathrm{~S}$ 422.224, Found 422.225.

(S)-Thioisobutyric acid S-[6-(biphenyl-3-ylcarbamoyl)-6-tert-butoxycarbonylaminohexyl]ester (14b): yellow oil; ${ }^{1} \mathrm{H}-\mathrm{NMR}\left(\mathrm{CDCl}_{3}, 500 \mathrm{MHz}, \delta\right.$; ppm) $8.24(1 \mathrm{H}$, broad s), $7.79(1 \mathrm{H}, \mathrm{s}), 7.58(2 \mathrm{H}, \mathrm{d}, J=7.6 \mathrm{~Hz}), 7.48$ $(1 \mathrm{H}, \mathrm{d}, J=7.9 \mathrm{~Hz}), 7.44-7.34(5 \mathrm{H}, \mathrm{m}), 4.98(1 \mathrm{H}, \mathrm{s}), 4.18(1 \mathrm{H}, \mathrm{s}), 2.84(2 \mathrm{H}, \mathrm{t}, J=7.3 \mathrm{~Hz}), 2.72(1 \mathrm{H}$, quintet, $J=$ 7.0 Hz), 2.00-1.95 (1H, m), 1.71-1.65 (1H, m), 1.60-1.54 (2H, m), 1.45-1.40 (13H, m), 1.18 (6H, d, J = 7.0 $\mathrm{Hz}) ;{ }^{13} \mathrm{C}-\mathrm{NMR}\left(\mathrm{CDCl}_{3}, 500 \mathrm{MHz}, \delta\right.$; ppm) 204.43, 170.44, 142.12, 140.66, 129.35, 128.73, 127.47, 127.20, 
123.16, 118.68, 81.28, 77.24, 43.13, 29.36, 28.33, 28.26, 25.16, 19.43; HRMS Calcd. for $\mathrm{C}_{28} \mathrm{H}_{38} \mathrm{O}_{4} \mathrm{~N}_{2} \mathrm{~S} 498.255$, Found 498.255 .

(S)-Thioisobutyric acid $S$-[6-tert-butoxycarbonylamino-6-(4-phenylthiazol-2-ylcarbamoyl)hexyl]ester (15b): yellow oil; ${ }^{1} \mathrm{H}-\mathrm{NMR}\left(\mathrm{CDCl}_{3}, 500 \mathrm{MHz}, \delta\right.$; ppm) 9.97 (1H, broad s), $7.82(2 \mathrm{H}, \mathrm{d}, J=7.0 \mathrm{~Hz}), 7.41$ (2H, t, $J=7.6 \mathrm{~Hz}), 7.32(1 \mathrm{H}, \mathrm{d}, J=7.3 \mathrm{~Hz}), 7.14(1 \mathrm{H}, \mathrm{s}), 5.05(1 \mathrm{H}, \mathrm{d}, J=7.5 \mathrm{~Hz}), 4.37(1 \mathrm{H}, \mathrm{s}), 2.83(2 \mathrm{H}, \mathrm{t}, J=7.3 \mathrm{~Hz})$, $2.73(1 \mathrm{H}$, quintet, $J=7.0 \mathrm{~Hz}), 2.00-1.95(1 \mathrm{H}, \mathrm{m}), 1.71-1.65(1 \mathrm{H}, \mathrm{m}), 1.60-1.54(2 \mathrm{H}, \mathrm{m}), 1.48(9 \mathrm{H}, \mathrm{s}), 1.45-1.40$ $(4 \mathrm{H}, \mathrm{m}), 1.18(6 \mathrm{H}, \mathrm{d}, J=7.0 \mathrm{~Hz}) ;{ }^{13} \mathrm{C}-\mathrm{NMR}\left(\mathrm{CDCl}_{3}, 500 \mathrm{MHz}, \delta ; \mathrm{ppm}\right) 204.42,170.36,157.27,150.08,134.34$, $128.72,128.05,126.12,107.95,80.94,77.24,54.61,43.12,31.75,29.29,28.30,28.20,25.00,19.42 ; \mathrm{HRMS}$ Calcd. for $\mathrm{C}_{25} \mathrm{H}_{37} \mathrm{O}_{4} \mathrm{~N}_{3} \mathrm{~S}_{2}$ 505.207, Found 505.213.

(S)-Thioisobutyric acid $S$-[6-tert-butoxycarbonylamino-6-(quinolin-3-ylcarbamoyl)hexyl]ester (16b): yellow oil; ${ }^{1} \mathrm{H}-\mathrm{NMR}\left(\mathrm{CDCl}_{3}, 500 \mathrm{MHz}, \delta\right.$; ppm) $8.76(1 \mathrm{H}, \mathrm{s}), 8.74(1 \mathrm{H}, \mathrm{s}), 8.03(1 \mathrm{H}, \mathrm{d}, J=8.8 \mathrm{~Hz}), 7.88(1 \mathrm{H}, \mathrm{d}$, $J=7.9 \mathrm{~Hz}), 7.62(1 \mathrm{H}, \mathrm{d}, J=7.2 \mathrm{~Hz}), 7.53(1 \mathrm{H}, \mathrm{d}, J=7.3 \mathrm{~Hz}), 5.01(1 \mathrm{H}, \mathrm{s}), 4.25(1 \mathrm{H}, \mathrm{s}), 2.85(2 \mathrm{H}, \mathrm{t}, J=7.5 \mathrm{~Hz})$, $2.73(1 \mathrm{H}$, quintet, $J=7.0 \mathrm{~Hz}), 2.04-1.95(1 \mathrm{H}, \mathrm{m}), 1.71-1.65(1 \mathrm{H}, \mathrm{m}), 1.60-1.54(2 \mathrm{H}, \mathrm{m}), 1.50-1.45(13 \mathrm{H}, \mathrm{m})$, $1.19(6 \mathrm{H}, \mathrm{d}, J=7.0 \mathrm{~Hz}) ;{ }^{13} \mathrm{C}-\mathrm{NMR}\left(\mathrm{CDCl}_{3}, 500 \mathrm{MHz}, \delta ; \mathrm{ppm}\right) 204.59,171.08,143.97,131.40,128.94,128.29$, 128.17, 127.74, 127.25, 123.88, 77.22, 43.14, 29.33, 28.31, 28.18, 25.13, 19.43; HRMS Calcd. for $\mathrm{C}_{25} \mathrm{H}_{35} \mathrm{O}_{4} \mathrm{~N}_{3} \mathrm{~S}$ 473.235, Found 473.234.

(S)-Thioisobutyric acid $S$-(6-tert-butoxycarbonylamino-6-cyclopentylcarbamoylhexyl)ester (17b).

Step 1: Preparation of (S)-(6-Bromo-1-cyclopentylcarbamoylhexyl)carbamic acid tert-butyl ester (23a). To a solution of $22(600 \mathrm{mg}, 1.85 \mathrm{mmol})$ obtained above in $N, N$-dimethylformamide $(4 \mathrm{~mL})$ were added 2-(1H-benzotriazol-1-yl)-1,1,3,3-tetramethyluroniumhexafluorophosphate (HBTU) (1.05 g, 2.78 mmol), 1-hydroxy-1H-benzotriazole monohydrate $\left(\mathrm{HOBt} \cdot \mathrm{H}_{2} \mathrm{O}\right)(283 \mathrm{mg}, 1.85 \mathrm{mmol})$, cyclopentylamine ( $275 \mathrm{uL}, 2.78$ mmol) and triethylamine $(650 \mathrm{uL}, 4.63 \mathrm{mmol})$, and the mixture was stirred at room temperature for $2 \mathrm{~h}$. The reaction mixture was poured into water and the whole was extracted with AcOEt. The AcOEt layer was separated, washed with $10 \%$ aqueous citric acid and brine, and dried over $\mathrm{Na}_{2} \mathrm{SO}_{4}$. Filtration, concentration in vacuo, purification by silica gel flash column chromatography (AcOEt $n$-hexane $=1 / 4)$ gave $276 \mathrm{mg}(38 \%)$ of 23a as a white solid: ${ }^{1} \mathrm{H}-\mathrm{NMR}\left(\mathrm{CDCl}_{3}, 500 \mathrm{MHz}, \delta\right.$; ppm) $5.93(1 \mathrm{H}, \mathrm{d}, J=6.7 \mathrm{~Hz}), 4.95(1 \mathrm{H}, \mathrm{s}), 4.17$ (1H, m), 
$3.95(1 \mathrm{H}, \mathrm{s}), 3.40(2 \mathrm{H}, \mathrm{t}, J=7.1 \mathrm{~Hz}), 1.98-1.89(2 \mathrm{H}, \mathrm{m}), 1.87-1.80(3 \mathrm{H}, \mathrm{m}), 1.67-1.58(5 \mathrm{H}, \mathrm{m}), 1.56-1.44(13 \mathrm{H}$, m), 1.39-1.36 (4H, m).

Step 2: Preparation of $(S)$-Thioisobutyric acid $S$-(6-tert-butoxycarbonylamino-6-cyclopentylcarbamoyl hexyl)ester (17b). Compound $\mathbf{1 7 b}$ was prepared from 23a obtained above using the same procedure as described for 12b (step 3) in 48\% yield: yellow oil; ${ }^{1} \mathrm{H}-\mathrm{NMR}\left(\mathrm{CDCl}_{3}, 500 \mathrm{MHz}, \delta\right.$; ppm) $5.97(1 \mathrm{H}, \mathrm{d}, J=7.6$ Hz), $4.97(1 \mathrm{H}, \mathrm{s}), 4.18(1 \mathrm{H}$, sextet, $J=6.9 \mathrm{~Hz}), 3.94(1 \mathrm{H}, \mathrm{s}), 2.83(2 \mathrm{H}, \mathrm{t}, J=7.2 \mathrm{~Hz}), 2.73(1 \mathrm{H}$, septet, $J=6.9$ Hz), $1.97-1.94(2 \mathrm{H}, \mathrm{m}), 1.83-1.78(1 \mathrm{H}, \mathrm{m}), 1.68-1.53(5 \mathrm{H}, \mathrm{m}), 1.44(9 \mathrm{H}, \mathrm{s}), 1.47-1.37(8 \mathrm{H}, \mathrm{m}), 1.19(6 \mathrm{H}, \mathrm{t}, J=$ $6.7 \mathrm{~Hz}) ;{ }^{13} \mathrm{C}-\mathrm{NMR}\left(\mathrm{CDCl}_{3}, 500 \mathrm{MHz}, \delta\right.$; ppm $) 204.29,170.59,155.72,77.23,50.37,43.11,35.04,34.95,29.40$, 28.43, 28.32, 28.30, 27.99, 25.06, 24.36, 19.42; Anal. Calcd. for $\mathrm{C}_{21} \mathrm{H}_{38} \mathrm{~N}_{2} \mathrm{O}_{4} \mathrm{~S} \cdot 1 / 3 \mathrm{H}_{2} \mathrm{O}: \mathrm{C}, 59.97 ; \mathrm{H}, 9.27$; , 6.66. Found: C, 59.91; H, 9.28; N, 6.79.

Compounds $18 b-20 b$ were prepared from 22 and the corresponding amines using the same procedure as described for $\mathbf{1 7 b}$.

(S)-Thioisobutyric acid $S$-(6-tert-butoxycarbonylamino-6-cyclohexylcarbamoylhexyl)ester (18b): yellow oil; ${ }^{1} \mathrm{H}-\mathrm{NMR}\left(\mathrm{CDCl}_{3}, 500 \mathrm{MHz}, \delta\right.$; ppm) $5.88(1 \mathrm{H}, \mathrm{d}, J=8.2 \mathrm{~Hz}), 4.99(1 \mathrm{H}, \mathrm{s}), 3.94(1 \mathrm{H}, \mathrm{s}), 3.75(1 \mathrm{H}, \mathrm{s}), 2.83$ $(2 \mathrm{H}, \mathrm{t}, J=7.4 \mathrm{~Hz}), 2.73(1 \mathrm{H}$, quintet, $J=7.0 \mathrm{~Hz}), 1.90-1.78(3 \mathrm{H}, \mathrm{m}), 1.72-1.68(2 \mathrm{H}, \mathrm{m}), 1.64-1.56(3 \mathrm{H}, \mathrm{m})$, 1.44 (9H, s), 1.39-1.28 (4H, m), 1.19-1.13 (8H, m); ${ }^{13} \mathrm{C}-\mathrm{NMR}\left(\mathrm{CDCl}_{3}, 500 \mathrm{MHz}, \delta ; \mathrm{ppm}\right) 204.35,170.87$, 155.69, 77.23, 48.13, 43.11, 33.04, 29.39, 28.41, 28.32, 25.49, 25.03, 24.72, 19.42; HRMS Calcd. for $\mathrm{C}_{22} \mathrm{H}_{40} \mathrm{O}_{4} \mathrm{~N}_{2} \mathrm{~S}$ 428.270, Found 428.276.

(S)-Thioisobutyric acid $S$-(6-tert-butoxycarbonylamino-6-cycloheptylcarbamoylhexyl)ester (19b): yellow oil; ${ }^{1} \mathrm{H}-\mathrm{NMR}\left(\mathrm{CDCl}_{3}, 500 \mathrm{MHz}, \delta\right.$; ppm) $5.98(1 \mathrm{H}, \mathrm{d}, J=8.2 \mathrm{~Hz}), 4.98(1 \mathrm{H}, \mathrm{s}), 3.95-3.91$ (2H, m), $2.83(2 \mathrm{H}, \mathrm{t}, J=7.3 \mathrm{~Hz}), 2.73(1 \mathrm{H}$, septet, $J=7.0 \mathrm{~Hz}), 1.88-1.87(2 \mathrm{H}, \mathrm{m}), 1.81-1.78(1 \mathrm{H}, \mathrm{m}), 1.61-1.36(20 \mathrm{H}$, m), $1.18(6 \mathrm{H}, \mathrm{d}, J=7.0 \mathrm{~Hz}) ;{ }^{13} \mathrm{C}-\mathrm{NMR}\left(\mathrm{CDCl}_{3}, 500 \mathrm{MHz}, \delta\right.$; ppm) 204.31, 171.16, 77.23, 60.40, 50.37, 43.11, 32.27, 29.40, 28.32, 28.30, 27.99, 25.06, 24.03, 21.06, 19.42, 14.21; Anal. Calcd. for $\mathrm{C}_{23} \mathrm{H}_{42} \mathrm{~N}_{2} \mathrm{O}_{4} \mathrm{~S} \cdot 1 / 2 \mathrm{H}_{2} \mathrm{O}: \mathrm{C}$, 61.16; H, 9.60; N, 6.20. Found: C, 62.23; H, 9.55; N, 6.32. 
(S)-Thioisobutyric acid $S$-(6-tert-butoxycarbonylamino-6-tert-butylcarbamoylhexyl)ester (20b): yellow oil; ${ }^{1} \mathrm{H}-\mathrm{NMR}\left(\mathrm{CDCl}_{3}, 500 \mathrm{MHz}, \delta\right.$; ppm) $5.80(1 \mathrm{H}, \mathrm{s}), 4.97(1 \mathrm{H}, \mathrm{s}), 3.88(1 \mathrm{H}, \mathrm{s}), 2.83(2 \mathrm{H}, \mathrm{t}, J=7.3 \mathrm{~Hz}), 2.73$ $(1 \mathrm{H}$, septet, $J=6.9 \mathrm{~Hz}), 1.81-1.74(1 \mathrm{H}, \mathrm{m}), 1.59-1.50(2 \mathrm{H}, \mathrm{m}), 1.48-1.40(11 \mathrm{H}, \mathrm{m}), 1.39-1.34(11 \mathrm{H}, \mathrm{m}), 1.18$

$(6 \mathrm{H}, \mathrm{d}, J=6.7 \mathrm{~Hz}) ;{ }^{13} \mathrm{C}-\mathrm{NMR}\left(\mathrm{CDCl}_{3}, 500 \mathrm{MHz}, \delta\right.$; ppm $) 204.29,171.11,77.23,51.32,43.11,32.33,29.41$, 28.71, 28.46, 28.31, 25.16, 19.42; Anal. Calcd. for $\mathrm{C}_{20} \mathrm{H}_{38} \mathrm{~N}_{2} \mathrm{O}_{4} \mathrm{~S} \cdot 2 / 3 \mathrm{H}_{2} \mathrm{O}$ : C, 57.96; H, 9.56; N, 6.76. Found: C, $57.86 ; \mathrm{H}, 9.29 ; \mathrm{N}, 6.89$.

(S)- (1-Cyclopentylcarbamoyl-6-mercaptohexyl)carbamic acid tert-butyl ester (17a).

Step 1: Preparation of (S)-Thioacetic acid S-(6-tert-butoxycarbonylamino-6-cycloheptylcarbamoyl hexyl)ester (24a). A solution of 23a (410 mg, $1.05 \mathrm{mmol}$ ) obtained above and KSAc (183 mg, $1.60 \mathrm{mmol})$ in $\mathrm{EtOH}(3 \mathrm{~mL})$ was stirred at room temperature for $24 \mathrm{~h}$. The reaction mixture was evaporated in vacuo. The residue was subjected to silica gel flash column chromatography $(\mathrm{AcOEt} / n$-hexane $=1 / 4)$ to give $303 \mathrm{mg}$ (75\%) of 24a as a yellow solid: ${ }^{1} \mathrm{H}-\mathrm{NMR}\left(\mathrm{CDCl}_{3}, 500 \mathrm{MHz}, \delta\right.$; ppm) $5.97(1 \mathrm{H}, \mathrm{d}, J=7.3 \mathrm{~Hz}), 4.97(1 \mathrm{H}, \mathrm{s})$, $4.18(1 \mathrm{H}, \mathrm{m}), 3.94(1 \mathrm{H}, \mathrm{m}), 2.85(2 \mathrm{H}, \mathrm{t}, J=7.3 \mathrm{~Hz}), 2.32(3 \mathrm{H}, \mathrm{s}), 1.97-1.96(2 \mathrm{H}, \mathrm{m}), 1.81-1.80(1 \mathrm{H}, \mathrm{m})$, $1.67-1.55(8 \mathrm{H}, \mathrm{m}), 1.44(9 \mathrm{H}, \mathrm{s}), 1.39-1.36(5 \mathrm{H}, \mathrm{m})$.

Step 2: Preparation of (S)-(1-Cyclopentylcarbamoyl-6-mercaptohexyl)carbamic acid tert-butyl ester (17a). To a solution of $\mathbf{2 4 a}(300 \mathrm{mg}, 0.778 \mathrm{mmol})$ obtained above in $\mathrm{EtOH}(5 \mathrm{~mL})$ was added $2 \mathrm{~N}$ aqueous $\mathrm{NaOH}(2 \mathrm{~mL}, 4.00 \mathrm{mmol})$, and the solution was stirred at room temperature for $10 \mathrm{~min}$. The reaction mixture was poured into water, and the whole was extracted with AcOEt. The AcOEt layer was separated, washed with water and brine, and dried over $\mathrm{Na}_{2} \mathrm{SO}_{4}$. Filtration, concentration in vacuo, and purification by silica gel flash column chromatography (AcOEt $/ n$-hexane $=1 / 4)$ gave $200 \mathrm{mg}(74 \%)$ of $\mathbf{1 7 a}$ as a white solid. The solid (106 mg) was recrystallized from $\mathrm{AcOEt} / n$-hexane to give $60.1 \mathrm{mg}$ of $\mathbf{1 7 a}$ as white crystals: $\mathrm{mp} 108-110{ }^{\circ} \mathrm{C}$; ${ }^{1} \mathrm{H}-\mathrm{NMR}\left(\mathrm{CDCl}_{3}, 500 \mathrm{MHz}, \delta\right.$; ppm) $5.93(1 \mathrm{H}, \mathrm{d}, J=7.0 \mathrm{~Hz}), 4.95(1 \mathrm{H}, \mathrm{s}), 4.19-4.16(1 \mathrm{H}, \mathrm{m}), 3.94(1 \mathrm{H}, \mathrm{s})$, $2.52(2 \mathrm{H}, \mathrm{q}, J=7.3 \mathrm{~Hz}), 1.98-1.96(2 \mathrm{H}, \mathrm{m}), 1.85-1.80(1 \mathrm{H}, \mathrm{m}), 1.67-1.52(7 \mathrm{H}, \mathrm{m}), 1.44(9 \mathrm{H}, \mathrm{s}), 1.42-1.31$ $(7 \mathrm{H}, \mathrm{m}) ;{ }^{13} \mathrm{C}-\mathrm{NMR}\left(\mathrm{CDCl}_{3}, 500 \mathrm{MHz}, \delta\right.$; ppm $)$ 171.89, 77.23, 48.04, 36.96, 33.79, 33.31, 28.65, 28.04, 25.68, 
25.56, 24.88, 24.56; Anal. Calcd. for $\mathrm{C}_{17} \mathrm{H}_{32} \mathrm{~N}_{2} \mathrm{O}_{3} \mathrm{~S}: \mathrm{C}, 59.27 ; \mathrm{H}, 9.36 ; \mathrm{N}, 8.13$. Found: $\mathrm{C}, 59.06 ; \mathrm{H}, 9.49 ; \mathrm{N}$, 7.97.

Compounds 18a and 19a were prepared from $24 \mathbf{b}$ and $24 \mathbf{c}$, respectively, using the same procedure as described for $17 \mathbf{a}$.

(S)-(1-Cyclohexylcarbamoyl-6-mercaptohexyl)carbamic acid tert-butyl ester (18a): mp 125-127 ${ }^{\circ} \mathrm{C}$; ${ }^{1} \mathrm{H}-\mathrm{NMR}\left(\mathrm{CDCl}_{3}, 500 \mathrm{MHz}, \delta\right.$; ppm) $5.85(1 \mathrm{H}, \mathrm{d}, J=7.9 \mathrm{~Hz}), 4.96(1 \mathrm{H}, \mathrm{s}), 3.95(1 \mathrm{H}, \mathrm{s}), 3.75(1 \mathrm{H}, \mathrm{s}), 2.51(2 \mathrm{H}$, q, $J=7.4 \mathrm{~Hz}), 1.90-1.78(3 \mathrm{H}, \mathrm{m}), 1.72-1.68(2 \mathrm{H}, \mathrm{m}), 1.64-1.56(4 \mathrm{H}, \mathrm{m}), 1.44(9 \mathrm{H}, \mathrm{s}), 1.43-1.31$ (7H, m), 1.19-1.13 (3H, m); ${ }^{13} \mathrm{C}-\mathrm{NMR}\left(\mathrm{CDCl}_{3}, 500 \mathrm{MHz}, \delta\right.$; ppm) 170.84, 77.23, 48.13, 33.74, 33.08, 32.93, 30.94, 28.33, 27.99, 25.49, 25.07, 24.71, 24.46; Anal. Calcd. for $\mathrm{C}_{18} \mathrm{H}_{34} \mathrm{~N}_{2} \mathrm{O}_{3} \mathrm{~S}: \mathrm{C}, 60.30 ; \mathrm{H}, 9.56$; N, 7.81. Found: C, 60.10; H, 9.51; N, 7.73; HRMS Calcd. for $\mathrm{C}_{18} \mathrm{H}_{34} \mathrm{O}_{3} \mathrm{~N}_{2} \mathrm{~S}$ 358.229, Found 358.229.

(S)-(1-Cycloheptylcarbamoyl-6-mercaptohexyl)carbamic acid tert-butyl ester (19a): $\mathrm{mp}$ 76-78 ${ }^{\circ} \mathrm{C}$; ${ }^{1} \mathrm{H}-\mathrm{NMR}\left(\mathrm{CDCl}_{3}, 500 \mathrm{MHz}, \delta\right.$; ppm) $5.92(1 \mathrm{H}, \mathrm{d}, J=7.6 \mathrm{~Hz}), 4.96(1 \mathrm{H}, \mathrm{s}), 3.95-3.91(2 \mathrm{H}, \mathrm{m}), 2.52(2 \mathrm{H}, \mathrm{q}, J$ $=7.3 \mathrm{~Hz}), 1.88-1.79(3 \mathrm{H}, \mathrm{m}), 1.62-1.58(7 \mathrm{H}, \mathrm{m}), 1.54-1.33(20 \mathrm{H}, \mathrm{m}) ;{ }^{13} \mathrm{C}-\mathrm{NMR}\left(\mathrm{CDCl}_{3}, 500 \mathrm{MHz}, \delta ; \mathrm{ppm}\right)$ 170.55, 77.21, 54.60, 50.38, 35.06, 34.96, 33.74, 32.34, 28.33, 27.99, 25.08, 24.47, 24.02; Anal. Calcd. for $\mathrm{C}_{18} \mathrm{H}_{34} \mathrm{~N}_{2} \mathrm{O}_{3} \mathrm{~S}: \mathrm{C}, 61.25 ; \mathrm{H}, 9.74 ; \mathrm{N}, 7.52$. Found: C, 61.14; H, 9.80; N, 7.43.

\section{Elemental Analysis}
Compd
calcd
found
$12 \mathrm{~b} \quad \mathrm{C}_{20} \mathrm{H}_{36} \mathrm{~N}_{2} \mathrm{O}_{6} \mathrm{~S} \cdot 1 / 4 \mathrm{H}_{2} \mathrm{O}$
C, 55.46; H, 6.00; N, 4.97
C, 55.21; H, 5.81; N, 5.21
17b $\mathrm{C}_{21} \mathrm{H}_{38} \mathrm{~N}_{2} \mathrm{O}_{4} \mathrm{~S} \cdot 1 / 3 \mathrm{H}_{2} \mathrm{OC}, 59.97 ; \mathrm{H}, 9.27 ; \mathrm{N}, 6.66$
C, 59.91; H, 9.28; N, 6.79
$19 \mathrm{~b} \quad \mathrm{C}_{23} \mathrm{H}_{42} \mathrm{~N}_{2} \mathrm{O}_{4} \mathrm{~S} \cdot 1 / 2 \mathrm{H}_{2} \mathrm{O}$
C, 61.16; H, 9.60; N, 6.20
C, 62.23; H, 9.55; N, 6.32 

$20 \mathrm{~b} \quad \mathrm{C}_{20} \mathrm{H}_{38} \mathrm{~N}_{2} \mathrm{O}_{4} \mathrm{~S} \cdot 2 / 3 \mathrm{H}_{2} \mathrm{OC}, 57.96 ; \mathrm{H}, 9.56 ; \mathrm{N}, 6.76$
C, 57.86; H, 9.29; N, 6.89
17a $\quad \mathrm{C}_{17} \mathrm{H}_{32} \mathrm{~N}_{2} \mathrm{O}_{3} \mathrm{~S}$
C, 59.27; H, 9.36; N, 8.13
C, 59.06; H, 9.49; N, 7.97
19a $\quad \mathrm{C}_{18} \mathrm{H}_{34} \mathrm{~N}_{2} \mathrm{O}_{3} \mathrm{~S}$
C, 61.25; H, 9.74; N, 7.52
C, 61.14; H, 9.80; N, 7.43

\section{Biology}

\section{Western Blot Analysis}

HCT116 human colon cancer cells were purchased from American Type Culture Collection (ATCC, Manassas, VA, U.S.A.) and cultured in McCoy5A culture medium containing penicillin and streptomycin, which was supplemented with fetal bovine serum as described in the ATCC instructions. HCT-116 cells (5 x $10^{5}$ ) were treated for $8 \mathrm{~h}$ with samples at the indicated concentrations in 10\% FBS-supplemented McCoy's 5A medium then collected and extracted with SDS buffer. Protein concentrations of the lysates were determined using a Bradford protein assay kit (Bio-Rad Laboratories); equivalent amounts of proteins from each lysate were resolved in 15\% SDS-polyacrylamide gel and then transferred onto nitrocellulose membranes (Bio-Rad Laboratories). After blocking for 30 min with Tris-buffered saline (TBS) containing 3\% skim milk, the transblotted membrane was incubated overnight at $4{ }^{\circ} \mathrm{C}$ with hyperacetylated histone $\mathrm{H} 4$ antibody (Upstate Biotechnology) (1:4000 dilution), acetylated $\alpha$-tubulin antibody (SIGMA) (1:4000 dilution) or $\beta$-actin antibody (Abcam) (1:500 dilution) in TBS containing 3\% skim milk. After probing with the primary antibody, the membrane was washed twice with water, then incubated with goat anti-rabbit or anti-mouse IgG-horseradish peroxidase conjugates (diluted 1:5000) for $2 \mathrm{~h}$ at room temperature, and further washed twice with water. The immunoblots were visualized by enhanced chemiluminescence. As for compound $\mathbf{1 7 b}$, the western blot band intensity was quantified using Scion Image software, Scion Corporation, USA. 


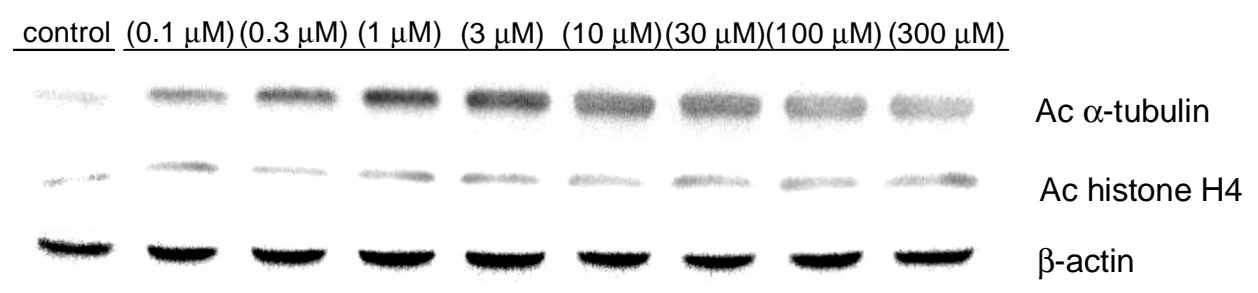

Figure 1S. Western blot detection of acetylated $\alpha$-tubulin and histone H4 levels in HCT116 cells treatment for $8 \mathrm{~h}$ with $\mathbf{1 7 b}$.

\section{Enzyme Assays}

The inhibitory activities of test compounds against partially purified HDAC1, HDAC4 and HDAC6 was assayed according to a method reported in Proc. Natl. Acad. Sci. U.S.A. 2001, 98, 87-92. 